ARTICLE

\title{
Immuno-genomic landscape of osteosarcoma
}

\author{
Chia-Chin Wu (1) 1,10, Hannah C. Beird (10) 1,10, J. Andrew Livingston (10 2,3,10, Shailesh Advani3 ${ }^{3}$, Akash Mitra (1) 1, \\ Shaolong $\mathrm{Cao}^{4}$, Alexandre Reuben (1) ${ }^{5}$, Davis Ingram ${ }^{6}$, Wei-Lien Wang ${ }^{6}$, Zhenlin Ju${ }^{4}$, Cheuk Hong Leung ${ }^{7}$, \\ Heather Lin7, Youyun Zheng1, Jason Roszik (1) 1, Wenyi Wang ${ }^{4}$, Shreyaskumar Patel ${ }^{2}$, Robert S. Benjamin², \\ Neeta Somaiah (1D 2 ${ }^{2}$, Anthony P. Conley², Gordon B. Mills ${ }^{8}$, Patrick Hwu², Richard Gorlick ${ }^{3}$, Alexander Lazar (1) ${ }^{6}$, \\ Najat C. Daw ${ }^{3,11}$, Valerae Lewis ${ }^{9,11}$ \& P. Andrew Futreal (1) 1,11凶
}

Limited clinical activity has been seen in osteosarcoma (OS) patients treated with immune checkpoint inhibitors $(\mathrm{ICl})$. To gain insights into the immunogenic potential of these tumors, we conducted whole genome, RNA, and T-cell receptor sequencing, immunohistochemistry and reverse phase protein array profiling (RPPA) on OS specimens from 48 pediatric and adult patients with primary, relapsed, and metastatic OS. Median immune infiltrate level was lower than in other tumor types where $\mathrm{ICl}$ are effective, with concomitant low T-cell receptor clonalities. Neoantigen expression in OS was lacking and significantly associated with high levels of nonsense-mediated decay (NMD). Samples with low immune infiltrate had higher number of deleted genes while those with high immune infiltrate expressed higher levels of adaptive resistance pathways. PARP2 expression levels were significantly negatively associated with the immune infiltrate. Together, these data reveal multiple immunosuppressive features of OS and suggest immunotherapeutic opportunities in OS patients.

\footnotetext{
${ }^{1}$ Department of Genomic Medicine, The University of Texas MD Anderson Cancer Center, Houston, TX, USA. ${ }^{2}$ Department of Sarcoma Medical Oncology, The University of Texas MD Anderson Cancer Center, Houston, TX, USA. ${ }^{3}$ Department of Pediatrics, The University of Texas MD Anderson Cancer Center, Houston, TX, USA. ${ }^{4}$ Department of Bioinformatics and Computational Biology, The University of Texas MD Anderson Cancer Center, Houston, TX, USA ${ }^{5}$ Department of Thoracic/Head \& Neck Medical Oncology, The University of Texas MD Anderson Cancer Center, Houston, TX, USA. ${ }^{6}$ Department of Pathology, The University of Texas MD Anderson Cancer Center, Houston, TX, USA. ${ }^{7}$ Department of Biostatistics, The University of Texas MD Anderson Cancer Center, Houston, TX, USA. ${ }^{8}$ Department of Systems Biology, The University of Texas MD Anderson Cancer Center, Houston, TX, USA. ${ }^{9}$ Department of Orthopedic Oncology, The University of Texas MD Anderson Cancer Center, Houston, TX, USA. ${ }^{10}$ These authors contributed equally Chia-Chin Wu, Hannah C. Beird, J. Andrew Livingston ${ }^{11}$ These authors jointly supervised this work. Najat C. Daw, Valerae Lewis, P. Andrew Futreal

凶email: afutreal@mdanderson.org
} 
O steosarcoma (OS) is the most common primary solid tumor malignancy of the bone predominantly occurring in adolescents with a second peak in incidence amongst older adults ${ }^{1}$. For patients presenting with localized disease at diagnosis, standard multi-agent chemotherapy combined with surgical resection yields long-term survival rates of $\sim 70 \%{ }^{1,2}$. Metastatic disease either at diagnosis or at the time of recurrence portends a poor prognosis with survival of $20-30 \%{ }^{3,4}$.

Recent whole genome sequencing (WGS) and molecular profiling studies undertaken in predominantly pediatric populations have shown high levels of chromosome structural variations, rearrangements resulting from chromothripsis (20-89\%) as well as mutation clusters known as kataegis (50-85\% of cases) that result in significant disease heterogeneity but few recurrent clinically actionable alterations ${ }^{5-7}$. These studies have yielded insights into aberrant signaling pathways such as $\mathrm{PI} 3 \mathrm{~K} / \mathrm{mTOR}$ $(24 \%)^{7}$, IGF signaling $(7 \%)^{6}$, and Wnt signaling 8 . However, the efficacy of targeted therapies such as mTOR inhibitors in unselected patient populations with relapsed osteosarcoma has been limited ${ }^{9,10}$. The degree of genomic instability suggests that the burden of antigens and neoantigens would provide an immunogenic potential in OS. However, this rationale has not met with the expected responses in current trials using immune checkpoint inhibitors in $\mathrm{OS}^{11,12}$. Currently, the population of interest for developing immunotherapy in OS are patients with poor risk disease (i.e., those with recurrence or metastasis following standard chemotherapy). Here, we use multi-platform profiling in parallel on such a population, including both children and adults, to define the immunogenic potential and to propose strategies to enhance the efficacy of immunotherapy.

\section{Results}

Our cohort consisted of patients with high-grade osteosarcoma with poor-risk ( $73 \%$ having unfavorable pathologic response to standard therapy) and adverse survival outcomes (67\% deceased) (Table 1, Supplemental Data 1). This cohort was enriched for relapsed $(13 / 54 ; 23 \%)$ and metastatic specimens $(27 / 54 ; 51 \%)$. We first assessed genomic complexity within the cohort by using high-depth whole genome sequencing (WGS) on 35 specimens with matched normal (average coverage for tumor and normal were $78 \mathrm{X}$ and $39 \times$, respectively) (Supplemental Data 2). These data were then integrated with transcriptome, T-cell receptor (TCR) sequencing, reverse phase protein array (RPPA), and immunostaining results to characterize the immunogenomic landscape of OS.

Genomic landscape. The genomic landscape in these largely advanced tumors was similar to what has been reported (Supplemental Fig. 1, Supplemental Data $3 a-b)^{5-7}$. No truncating germline mutations associated with DNA repair were observed. In addition, no significant differences were seen across the primary, local recurrence, and lung metastasis specimens in mutation burden (based on nonsilent single nucleotide variants, small insertions and deletions), predicted neoantigens, subclonal proportions, copy number alterations, and altered pathways (Supplemental Fig. 2, Supplemental Data 4a-b). Nonetheless, we identified several interesting genomic features in our cohort. First, the contribution of mutation signature 8 (Supplemental Fig. 3a) was positively associated with mutation burden, disease relapse status, and survival, but not with kataegis (Fig. 1a, b). Second, the high levels of genomic rearrangements (Supplemental Fig. 1e) could be divided into two major groups corresponding to rearrangement signatures 2 (non-clustered) and 4 (clustered) (Fig. 1c) ${ }^{13}$. There was a trend for younger patients to have rearrangements that are clustered and associated with chromothripsis

\section{Table 1 Clinical characteristics of $\mathbf{4 8}$ patients with osteosarcoma.}

\section{Characteristic}

n (\%)

Median age at diagnosis in years (range)

$27(5-81)$

Gender

Male

Female

29 (60)

Race

White

19 (40)

Hispanic

30 (63)

Black

10 (21)

Asian

5 (10)

Asian

3 (6)

Localized

37 (77)

Metastatic

11 (23)

Histologic subtype

Osteoblastic

14 (29)

Fibroblastic

9 (19)

Chondroblastic

6 (13)

Extraskeletal

6 (13)

Dedifferentiated parosteal

5 (10)

Telangiectatic

2 (4)

Giant cell rich

1 (2)

High-grade surface

$1(2)$

Other-high grade

4 (8)

Pathologic response to neoadjuvant chemotherapy ( $n=41$ patients)

Good (tumor necrosis $\geq 90 \%$ )

$11(27)$

Poor (tumor necrosis $<90 \%$ )

30 (73)

Vital status

Alive

16 (33)

Dead

32 (67)

Specimen type $(n=54)^{\star \star}$

Primary tumor

$14(26)$

Local recurrence

13 (23)

Metastasis

27 (51)

Testing platforms (number of specimens) ${ }^{\star \star}$

Whole genome sequencing (WGS)

RNA sequencing (RNAseq)

Reverse phase protein prray (RPPA)

$\mathrm{T}$-cell receptor sequencing (TCR)

Immunohistochemistry

35

51

38

41

33

* For patients who received neoadjuvant chemotherapy

${ }^{\star}$ Two samples from same tumor site in 3 patients

as compared with older patients, which was also observed in the Therapeutically Applicable Research to Generate Effective Treatments (TARGET) cohort that is composed of mostly pediatric OS (Fig. 1d, $P<0.01$, Wilcoxon rank sum test; Supplemental Fig. 3b). On average, $18.9 \%$ of breakpoints occurred in fragile sites, which is comparable to the mean found in the BOCA-UK cohort (16.5\%) (Supplemental Data 5). Third, OS cells may maintain viability of their TP53/RB1 mutation-induced unstable genomes through whole-genome doubling (WGD) or telomere lengthening6,14,15 (Supplemental Fig. 3c). In our cohort, both WGD and normalized telomere lengths had significant positive association with high copy number and rearrangement burden (Fig. 1 e $P<0.001, P<1$ e4, respectively, Pearson's correlation). Up to $50 \%(18 / 36)$ of patients had losses of heterozygosity in TP53 and/or RB1 along with WGD (Supplemental Fig. 1a). Given the inherent lower likelihood of losing two copies after WGD, this supports that TP53 and $R B 1$ aberrations likely occurred before WGD ${ }^{16,17}$. Genetic alterations and expression of TERT do not have significantly longer telomeres (Supplemental Fig. 3d). Instead, lower expression levels of ATRX were significantly correlated with longer normalized telomere lengths (Fig. 1f). Seven patients with deleterious alterations in $A T R X$ as well as one patient with copy number loss 
a

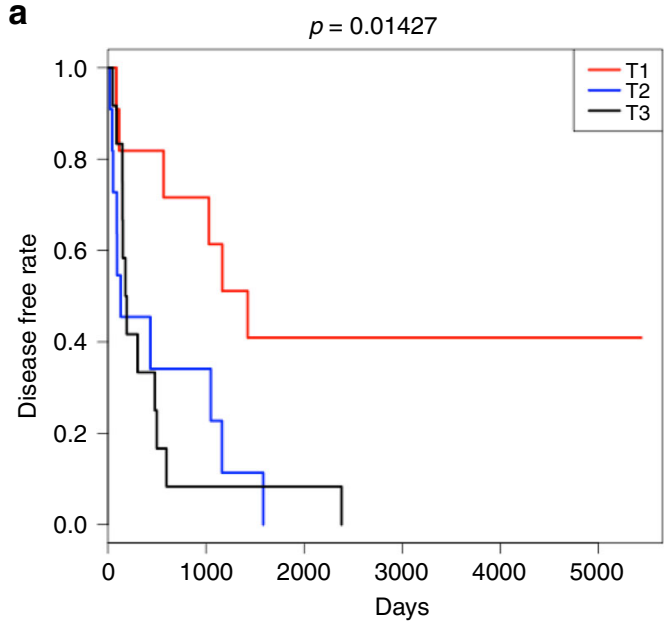

C

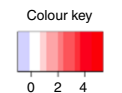

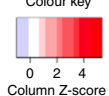

$$
\text { Chromothtripsis }
$$

Specimen types

$$
\begin{aligned}
& \begin{array}{l}
\quad \text { Spe } \\
\square \text { Age }<18 \\
18<=\text { Age }
\end{array} \\
& \text {-18<= Age }<50 \\
& \text { - Age }>=50
\end{aligned}
$$
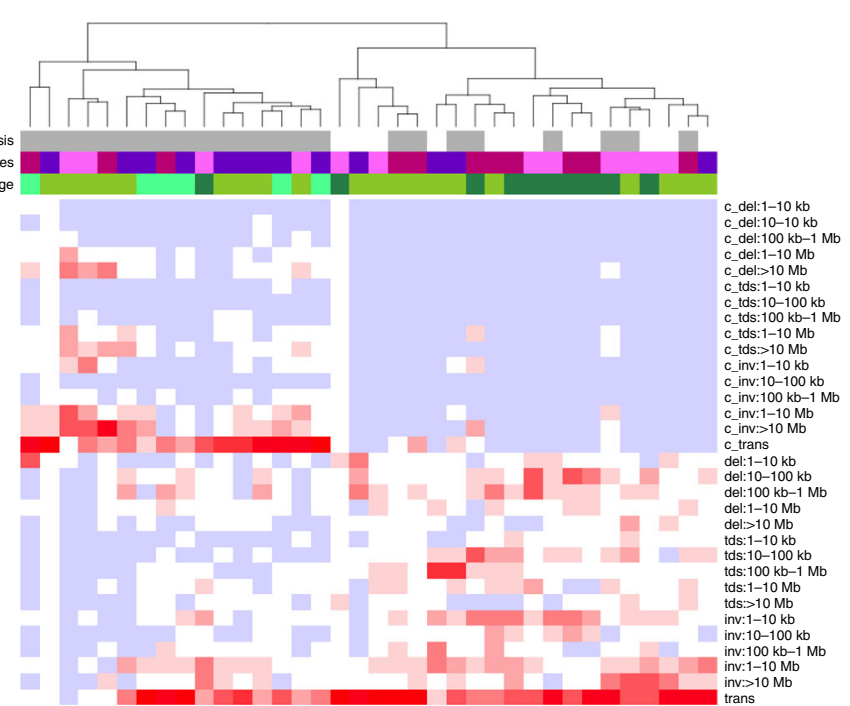

b

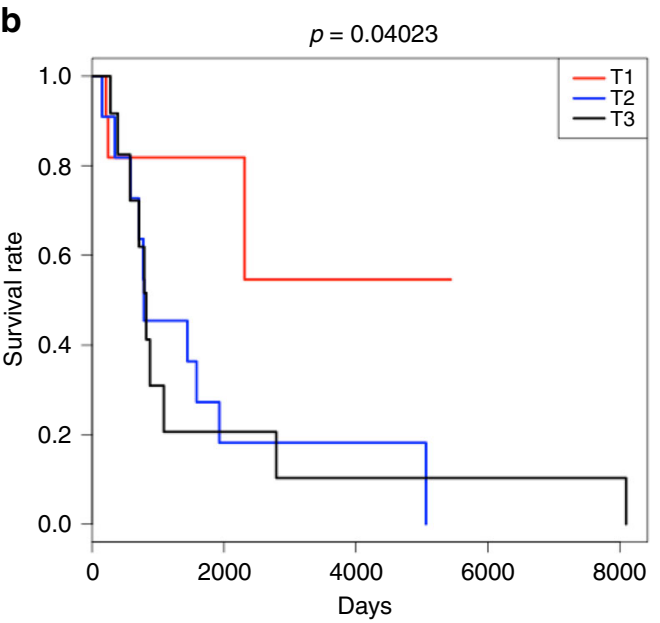

d

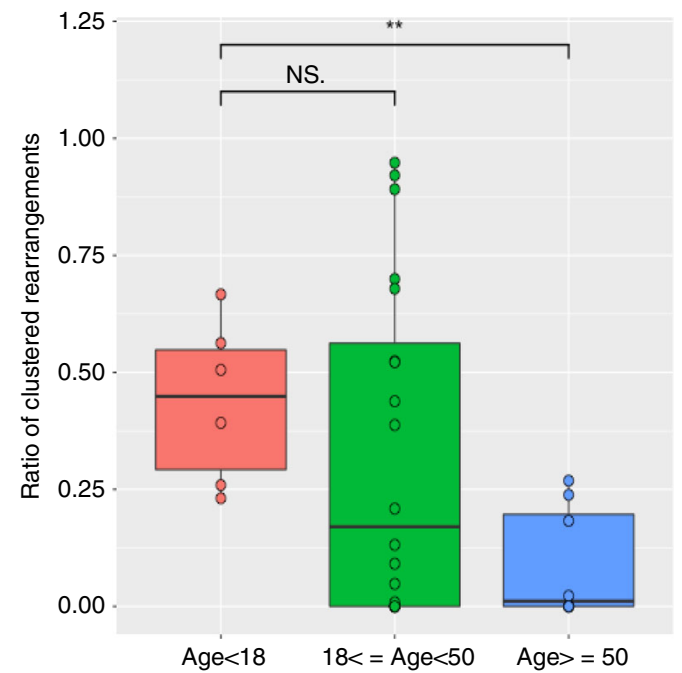

e

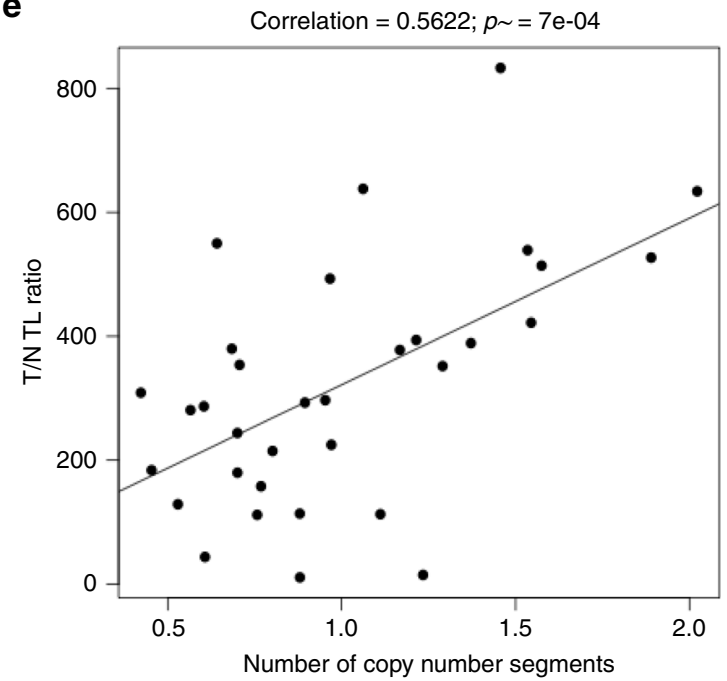

f

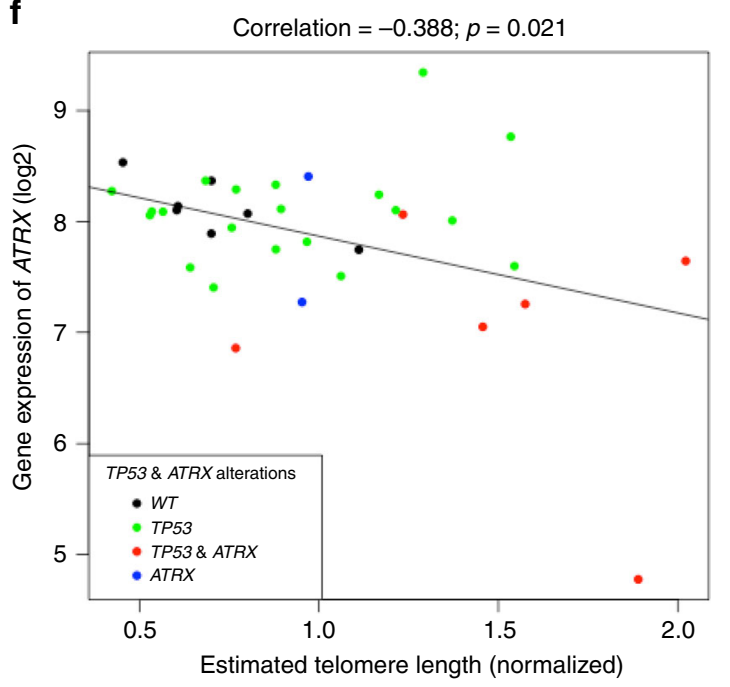

in DAXX had telomere lengths greater than the cohort median (Fig. 1f). Patient samples with the longest telomere length carried alterations in both TP53 and ATRX, supporting the permissive context in which TP53 alterations can allow for activation of alternative lengthening of telomeres (ALT) ${ }^{18}$. In addition, the expression levels of telomere maintenance genes HNRNPA2B1,
WRN, and $H U S 1^{19}$, were also significantly correlated with telomere length $(P<0.001, P<0.05, P<0.05$, respectively, Pearson's correlation) (Supplemental Data 6). However, the exact mechanisms surrounding telomere maintenance in the ALT pathway is unclear. Thus, effect of the telomere-related mutations on ALT are yet to be explored. 
Fig. 1 Clinical associations with somatic alterations in OS. a, b Kaplan-Meier survival analysis (disease-free survival for $\mathbf{a}$; overall survival for $\mathbf{b}$ ) of mutation signature 8 scores which describe the contribution of mutation signature 8 to the point mutation profile. The cohort was separated into tertiles based on signature 8 scores. Group 1 (denoted as T1) is the first tertile with lowest mutation signature 8 scores. Groups 2 and 3 (denoted as T2 and T3 respctively) are the next 2 tertiles with higher mutation signature 8 scores. c Hierarchical clustering heatmap of rearrangements classification pattern identified in our cohort along with clinical features: age of diagnosis ( $<18$ years, $18-<50$ years, and $>50$ years), tumor specimen type (primary, local recurrence, metastasis), and vital status (dead, alive). Structural rearrangements were classified based on their type: deletion (del), tandem duplication (tds), inversion (inv), and interchromosomal translocation (trans), and size, and clustered (denoted as c_) versus non-clustered events. $\mathbf{d}$ Boxplot showing the ratio of clustered rearrangements within associated with chromothriptic regions as compared across three groups of specimens based on age of diagnosis ( $<18$ years, $18-<50$ years, and $>50$ years). Then significance values ( $P$-values) of the comparisons are from the Wilcoxon rank sum test are shown in asterisks. e Pearson correlation between normalized telomere length and number of copy number segments of samples. $\mathbf{f}$ Pearson correlation between normalized telomere length and ATRX gene expression level (in log2 scale) of samples. Samples with both ATRX and TP53 alterations, ATRX alterations alone, and TP53 alterations alone were respectively marked as red, blue, and green color.
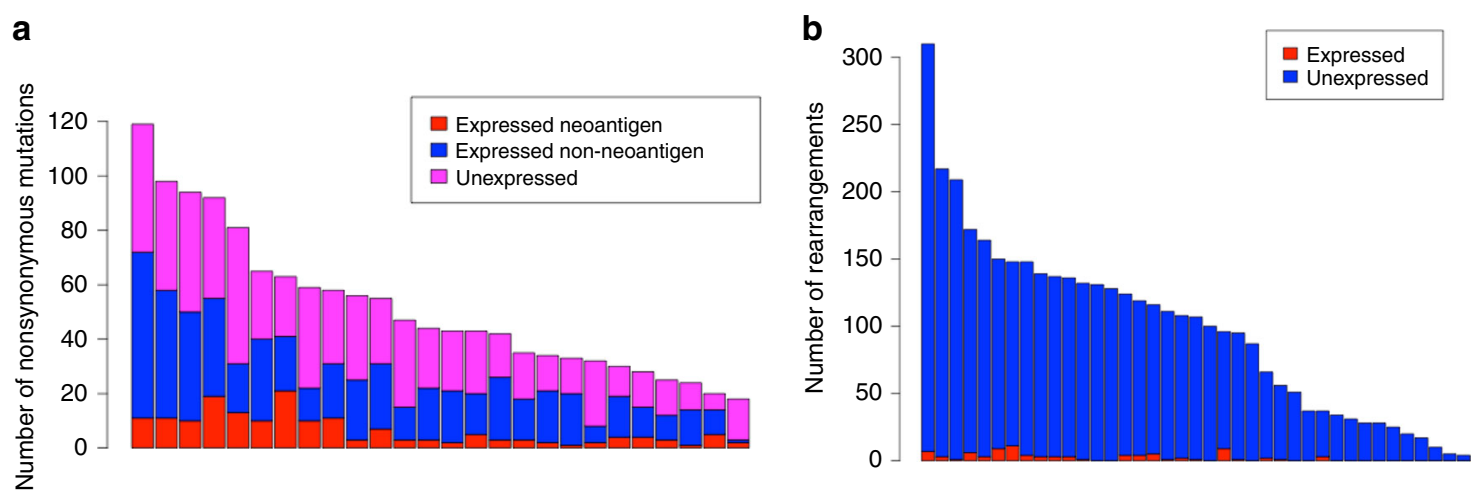

Fig. 2 Expressed neoantigens and rearrangements. a Stacked bar chart for number of nonsynonymous mutations and neoantigens detected by WGS and capture-based RNASeq. (expressed neoantigen: nonsynonymous mutations found in both WGS and capture-based RNASeq that are predicted to be a neoantigen; expressed non-neoantigen: nonsynonymous mutations found in both WGS and capture-based RNASeq that are not predicted to be a neoantigen; unexpressed: found in WGS only). b Overlapping rearrangements between WGS and RNASeq based rearrangements where both partners are within coding regions (unexpressed: found in WGS only; expressed:found in both WGS and RNASeq).

Furthermore, we also compared genomic features based upon pathologic tumor response to neoadjuvant chemotherapy as well as a comparison across histologic subtypes. We found that samples with favorable tumor necrosis (defined as $\geq 90 \%$ necrosis following chemotherapy) have higher COSMIC 3 signature scores, telomere lengths, and Th2 T cell scores as compared to those with poor tumor necrosis $(<90 \%)$ (Supplemental Data 7$)$. This suggests that the genomes may be less stable and chronic activation of immune response in those with favorable necrosis ${ }^{20}$.

Mutation burdens were not associated with immune infiltrate levels. The high levels of genomic rearrangements and moderate point mutation burdens in OS suggests that the levels of neoantigens should be high enough to elicit an immune response ${ }^{21}$. Although higher nonsilent mutation burden was associated with higher number of predicted neoantigens in our cohort (Supplemental Fig. 4a), transcriptome immune infiltrate scores (ESTIMATE $)^{22}$ were not influenced by having more neoantigens (Supplemental Fig. 4b). This was further supported by the lack of association between an RPPA immune-related markers (Caspase7 (cleaved D198), Lck, Syk, and Pded-1L1) (see Methods) and predicted neoantigen burden (Supplemental Fig. 4c). Similarly, no relationship was found between immune infiltrate scores (ESTIMATE) and the total number of rearrangements (Supplemental Fig. 4d).

Low level of predicted neoantigen expression. Given the levels of rearrangements and mutation burden, we explored whether these genomic alterations are expressed and the potential levels of neoantigens. Fewer than $30 \%$ of expressed nonsynonymous changes as detected by RNAseq were predicted to be strong-binding neoantigens (5-30\% per patient) (Fig. 2a). As expected, unexpressed mutations tended to occur in genes that have low expression (Supplemental Fig. 5a) or whose variant allele frequencies were $<0.25$, likely a limit in sensitivity due to RNASeq (Supplemental Fig. 5b). Limited overlap between point mutations identified in both DNA and RNA sequencing has been observed in non-small cell lung cancer as well as glioblastoma suggesting that this finding is not unique to $\mathrm{OS}^{23,24}$. In addition, few predicted rearrangements involving coding regions were expressed (Fig. 2b). Of interest, we observed a positive association between nonsense- mediated decay (NMD) factors and the number of gene-containing rearrangements as well as immune scores $(P<0.05$, Pearson's correlation) (Supplemental Fig. 6), suggesting that there may be substantial transcript suppression in rearranged OS genomes. Overall, despite substantial proportions of predicted neoantigenic mutations and genic rearrangements, there are few alterations ultimately expressed at high levels.

T-cells present but low $\mathrm{T}$-cell receptor productive clonality. The majority of specimens $(21 / 33,64 \%)$ showed medium or high density staining for $\mathrm{CD} 3+\mathrm{T}$-cells, which indicated that T-cell infiltrate was present in a most samples (Supplemental Data 8a). Lung metastases had higher CD3, CD4 T-cell and M2 macrophage $(\mathrm{CD} 163)$ staining densities than primary specimens $(P<$ $0.05, P<0.01$, and $P<0.05$, respectively, Wilcoxon rank sum test) (Supplemental Data 8b-c). However, T-cell activity as estimated by productive clonality was low overall (average $=0.09$ ) with a maximum of 0.3 in comparison to normal skin $(0.15)^{25}$ and healthy adult female peripheral blood mononuclear cells 
$(\sim 0.05-0.2)^{26}$ (Supplemental Data 8d). Five cases (WGS_12-15, WGS_12-23, WGS_12-24, WGS_12-35, WGS_12-7) had one clone that represented the largest proportion $(0.01-1)$ of the Tcell population (as measured by homeostatic space) and all of these had low numbers of T-cell clonotypes $(<100)$ (Supplemental Fig. 7). These data suggest a lack of T-cell clonal diversity that may blunt the ability to mount an effective immune response.

Insufficient immune infiltrate. To understand the immune infiltrate level of OS in a broader context, we compared immune infiltration scores (ESTIMATE) from our cohort against other tumor types profiled in The Cancer Genome Atlas (TCGA), the OS samples from the International Cancer Genome Consortium (ICGC) and TARGET studies, as well as four additional patients with metastatic OS who were treated with combination CTLA-4 blockade and PD-L1 blockade but exhibited no objective responses (Fig. 3a). The median immune scores from the ICGC cohort, TARGET, and the four pre-treatment OS samples for patients treated with ICI were comparable to our cohort. Skin cutaneous melanoma (TCGA-SKCM), lung cancer (TCGALUAD and TCGA-LUSC) and renal clear cell carcinoma (TCGAKIRC), are tumor types that have clinical benefit and response to immune checkpoint blockade related to high immune infiltrate levels whereas other tumor types are known to have very low immune infiltrate such as low-grade glioma (TCGA-LGG), prostate cancer (TCGA-PRAD), and uveal melanoma (TCGAUVM) and have seen limited activity with current immunotherapy approaches. Within this context, we observed that specimens from our cohort have intermediate median ESTIMATE scores that are lower than melanoma and lung cancer, but higher than uveal melanoma. When compared to other sarcoma subtypes, the median immune score of dedifferentiated liposarcoma (TCGA-DDLPS) and undifferentiated pleomorphic sarcoma (TCGA-UPS) - 2 soft tissue sarcoma subtypes where ICI are active-are higher than OS samples. We also examined samples whose ESTIMATE immune scores are in the highest quartile among all the samples, which may be likely benefit from the ICI. Overall, $8 \%(4 / 51)$ of our cohort samples, $10 \%(1 / 10)$ of the ICGC cohort, $15 \%(12 / 84)$ of the TARGET cohort, $0 \%(0 / 4)$ OS patients treated with ICI are in the highest quartile (Supplemental Fig. 8). These data indicate that most of OS specimens we examined may have insufficient immune infiltrate to elicit meaningful responses to ICI alone.

Evidence for multiple immuno-modulatory mechanisms. To characterize and compare the composition of the immune infiltrate across our samples, we first generated single sample GSEA gene expression enrichment scores for various immune cell types for each patient (based on gene lists from Charoentong et al. ${ }^{27}$. Hierarchical clustering on these scores revealed three clusters: $\mathrm{C} 1$, $\mathrm{C} 2$, and C3 with increasing levels of immune infiltrate: $\mathrm{C} 1$ having the lowest and C3 having the highest levels of all immune cell types including CD8 lymphocytes (Fig. 3b, Supplemental Fig. 9a). These significantly different levels of expression were also reflected in the immune infiltrate scores that were present (by ESTIMATE and TIMER) ${ }^{28}$ (Fig. 3c, Supplemental Fig. 9b-h) as well as cytolytic scores ${ }^{29}$ (GZMA and PRF1) (Fig. 3d). The TARGET dataset also revealed several clusters, two of which are comparable to our C1 "cold" and C3 "hot" immune infiltrate groups (Supplemental Fig. 10). For our cohort, 9 of the 14 older patients (age $>50$ ) were in the C3 group with higher immune infiltrate levels (Fig. 3b). This finding corresponded with the significantly higher CD8 + T-cell immunostaining that was orthogonally demonstrated amongst older patients $(P<0.01$, Wilcoxon rank sum test) (Supplemental Data 9a-b).
We then interrogated whether tumor-intrinsic immunosuppressive pathways were enriched between the "cold" $\mathrm{C} 1$ cluster and the relatively "hot" C3 cluster. Among those enriched deregulated pathways identified were: PD1 signaling, CTLA4 pathway, IFNG signaling, and others (Fig. 3e). This suggests that C3 tumors are actively enhancing expression of signals that inhibit T-cell activation (PD-L1, CTLA4, IFNG) as well as molecules such as IDO1 that participate in the recruitment of immunosuppressive cells. Although TCR productive clonality measures were higher in $\mathrm{C} 3$ than in $\mathrm{C} 1$, they were still low overall (Supplemental Fig. 9i). The lack of high TCR productive clonality amongst C3 may be due to adaptive immune resistance mechanisms such as higher PD-L1, CTLA4, IDO1 expression levels and the presence of myeloid-derived suppressor cells (MDSCs) in the C3 group as compared to C1 (Fig. 3e, f). For C1, the lack of TCR productive clonality could be explained in part by the lower levels of HLA antigen-presenting genes than seen in C3 (Supplemental Data 8e) and the number of deleted genes.

Recent studies have shown that high levels of genome aneuploidy in cancer are associated with lower levels of immune-related markers ${ }^{30,31}$. Indeed, higher number of copy number losses correlated with lower ESTIMATE immune infiltrate scores $(P<0.05$, Pearson's correlation), confirmed by the TARGET cohort (Fig. $4 \mathrm{a}, \mathrm{b}$ ). Also, specimens in $\mathrm{C} 1$ had a significantly higher number of deleted genes (Supplemental Fig. 10a). For the TARGET cohort, the number of genes with copy number gain were also negatively correlated with ESTIMATE immune scores whereas that was not seen in our cohort (Supplemental Fig. 11b). No significant relationship was observed among the three clusters in mutation burdens, numbers of predicted neoantigens, and numbers of rearrangements. A separate multiple regression analyses comparing genomic instability factors (number of losses, gains, rearrangements, somatic point mutations) and immune score revealed that losses had the greatest association with immune score $(P<0.01$, multiple linear regression). To identify genes with genomic alterations that were significantly associated with immune infiltrate, we applied an integration analysis of genomic alterations and ESTIMATE immune infiltrate scores. As the top hit for copy number losses, both TP53 loss and expression were significantly negatively correlated with ESTIMATE immune scores (See Methods and Supplemental Data 10b). Neither PTEN loss nor expression were associated (Supplemental Data 10a-c), contrary to what was found by Roh et al. in melanoma ${ }^{31}$. Thus, tumor type-specific associations may exist. Interestingly, the gene expression (Fig. 4c) and copy number gain of PARP2 (Fig. 4d), a druggable target, are both significantly negatively associated with the ESTIMATE immune score. This relationship between PARP2 expression and immune score was recapitulated in the TARGET dataset (Supplemental Fig. 11c). Thus, taken together, these data indicate that there are likely multiple immune-suppressive mechanisms in play in OS.

\section{Discussion}

We conducted a comprehensive genomic and immune characterization of post-treatment primary, local recurrence, and metastasis OS specimens from a cohort of poor prognosis patients to determine molecular bases for the lack of response of OS to immune checkpoint therapy. We observed very few differences in the genomic landscapes and expression profiles across the three groups of samples. However, several interesting genomic features were identified in our cohort. First, the enrichment in focal clustered rearrangements amongst younger patients suggests that oncogenesis may be more driven by catastrophic chromothripsis events in young OS patients as compared to older adults. Second, 
a

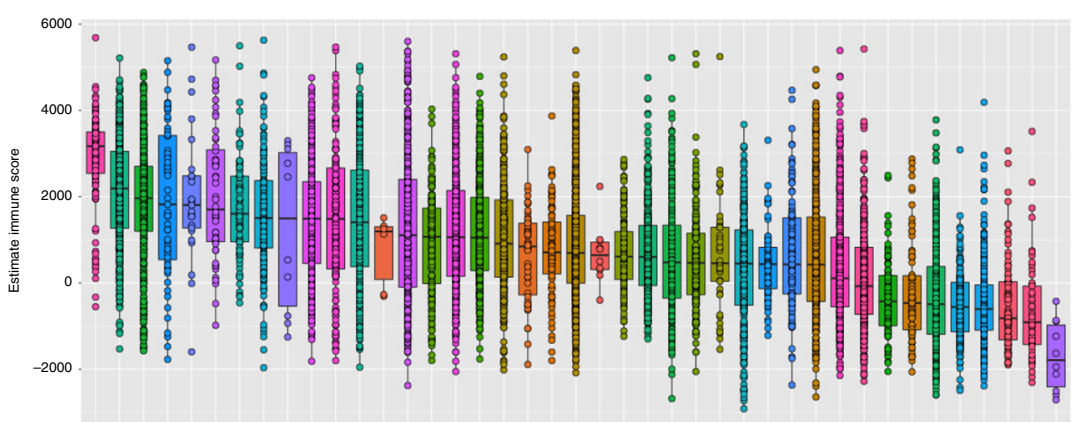

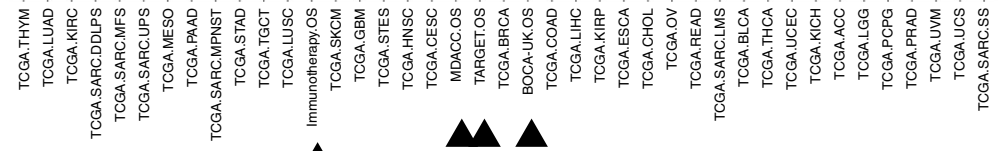

b

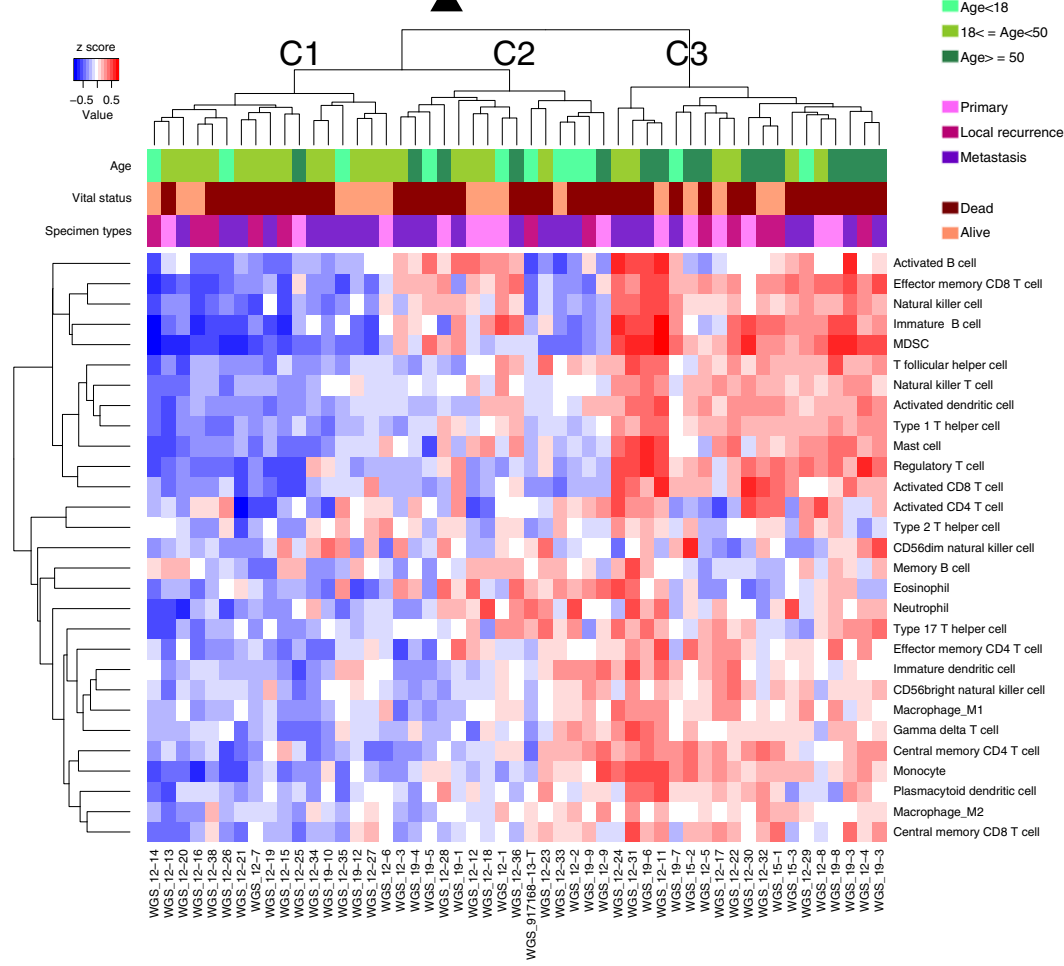

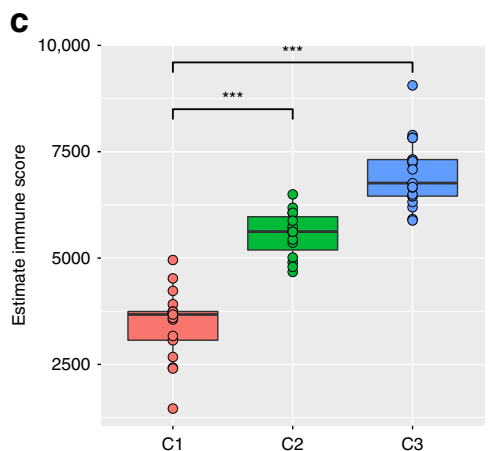

d

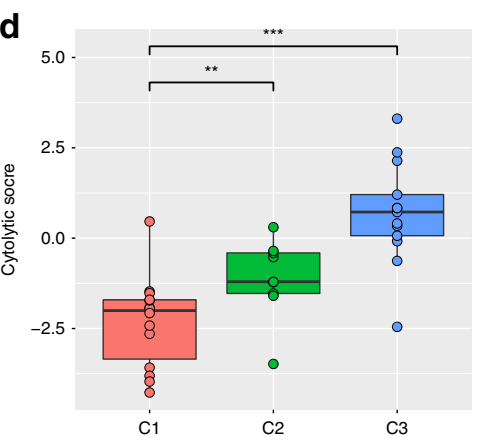

e

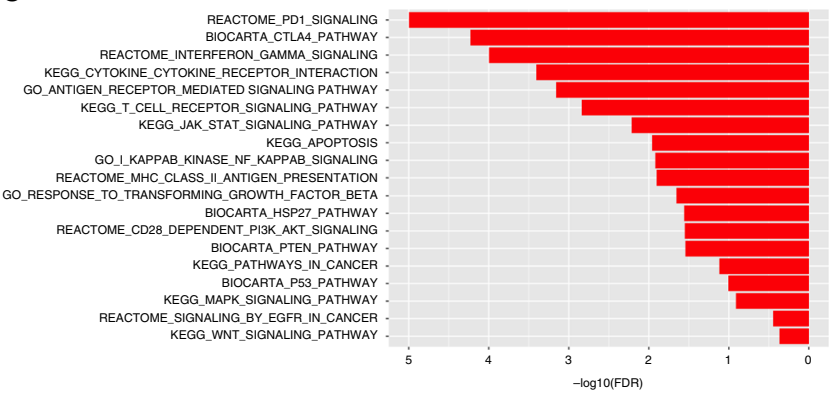

our data also suggests that there is a temporal order to loss of TP53 and subsequent ALT and WGD events to promote genome instability in OS. The role of ALT in genomic instability may provide a therapeutic target in OS, as proposed by Flynn et al. ${ }^{32}$. In addition, we also identified enrichment for mutation signatures 5 and 8 as Behjati et al. ${ }^{6}$, and found signature 8 is significantly associated with worse prognosis. Mutation signature 3 was the predominant mutational signature enriched in the TARGET f

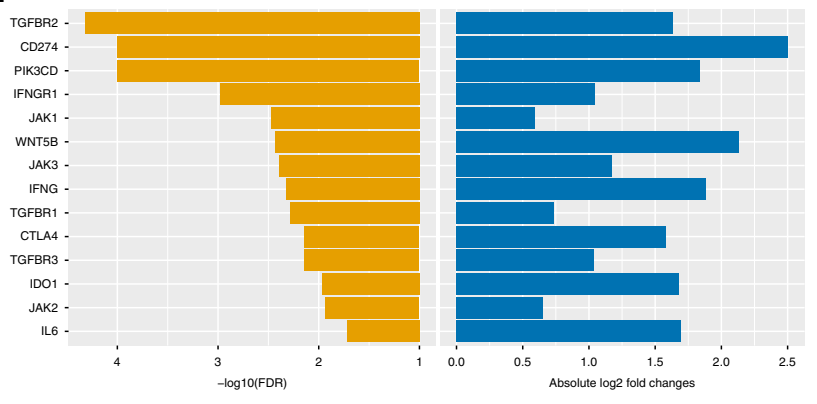

cohort $^{33}$. This discrepancy may be due to patient age (our cohort has more older patients) or that this enrichment is prevalent in particular subsets of OS that are yet to be defined.

We defined three broad immune subsets $(\mathrm{C} 1, \mathrm{C} 2, \mathrm{C} 3)$ with low, intermediate, and high levels of immune infiltrate-so called "hot" and "cold" tumors. Although the level of immune infiltrate and activity is high in a subset of OS (C3), an ineffective immune response may be due to the lack of neoantigens or to the presence 
Fig. 3 Immune profiling. a ESTIMATE immune scores in TCGA tumor types, our OS cohort (MDACC.OS), the OS cohort from ICGC (BOCA-UK.OS), the OS cohort from TARGET (TARGET.OS), and from four patients who underwent combination CTLA-4 blockade and PD-L1 blockade but exhibited no objective responses (Immunotherapy.OS). Note that TCGA-THYM is derived from the thymus made up mostly of lymphocytes and would have inflated scores. b. Unsupervised hierarchical clustering based on ssGSEA enrichment scores of each immune gene list from Charoentong et al. The three predominant clusters are referred to as " $\mathrm{C} 1$ " and "C2", and "C3" immune infiltrate groups. c Boxplot of the ESTIMATE immune scores of samples across the three immune clusters $\mathrm{C} 1, \mathrm{C2}$, and C3. The significances of the comparisons were from the Wilcoxon rank sum test are shown in asterisks. $\mathbf{d}$ The geometric mean expression of GZMA and PRF1 genes (cytolytic score) shown as a boxplot across the three immune clusters $\mathrm{C1}, \mathrm{C2}$, and C3. The significances of the comparisons were from the Wilcoxon rank sum test. e Immunosuppressive pathways that are significantly deregulated between immune clusters $\mathrm{C} 1$ and C3. The significance (FDR) of deregulation were from the GSEA analysis. $\mathbf{f}$ Fold change and significance of expression difference between C1 and C3 for key genes from pathways in e: CD274(PD-L1), CTLA4, IDO1, IFNG, IFNGR1, and IL6 (these genes were not present in the gene lists used to define these immune groups). The linear mode in the limma R package was used to determine significance.

a

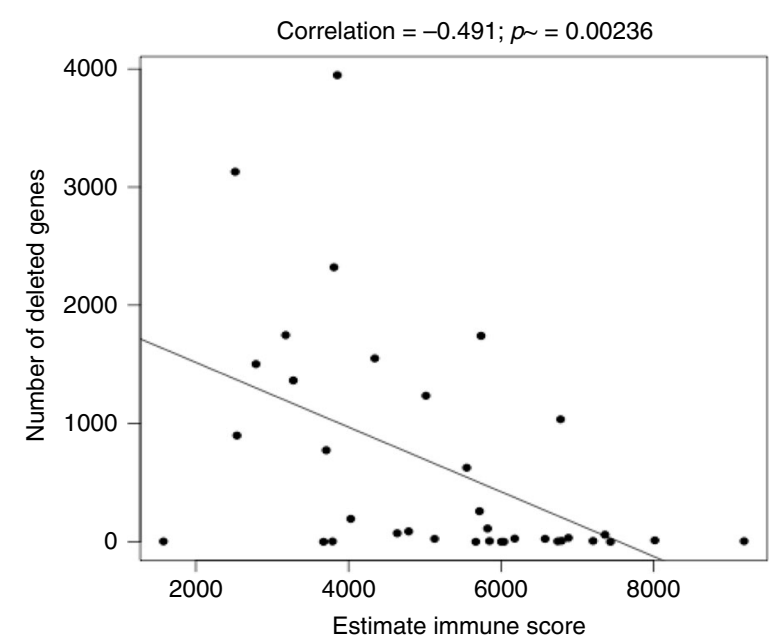

C

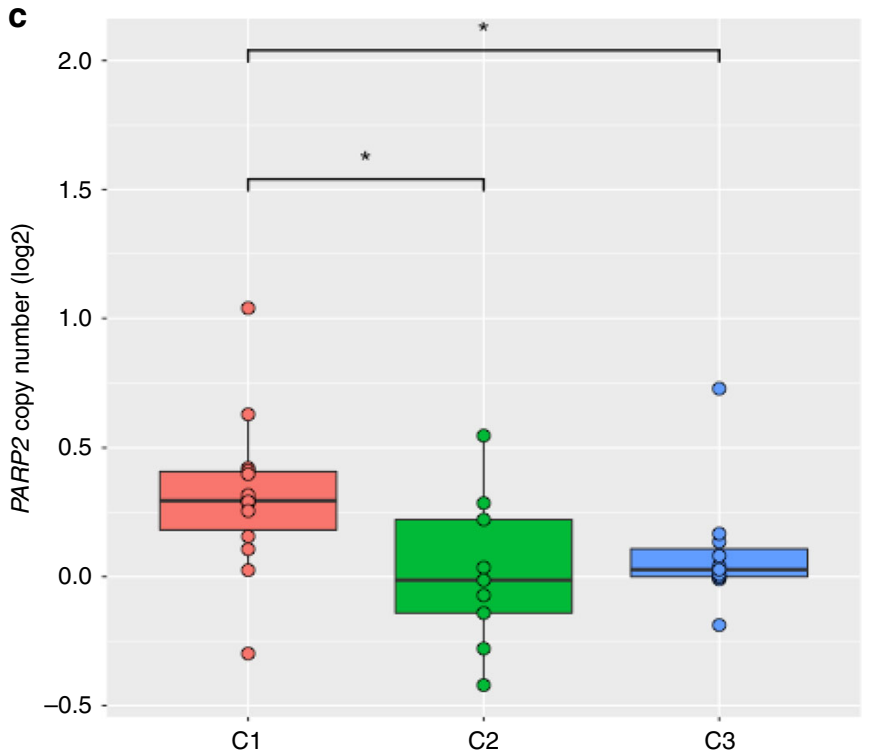

b

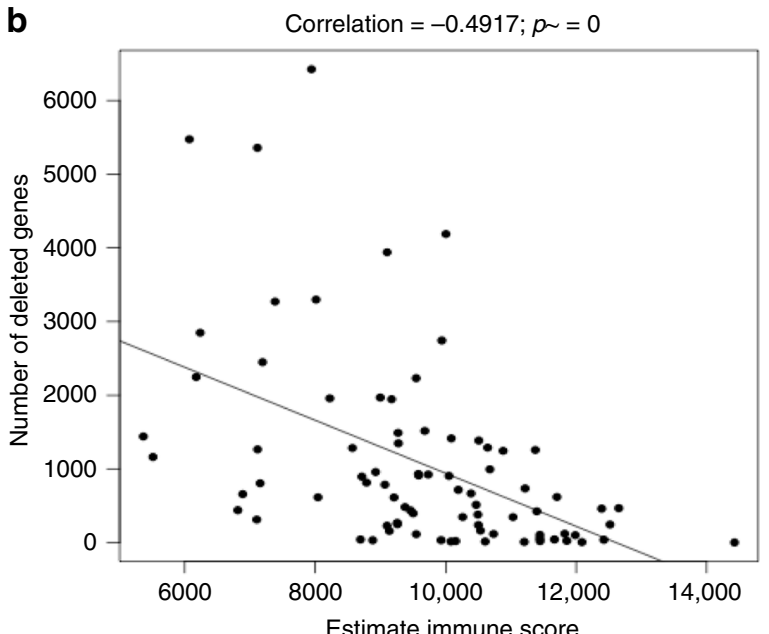

d
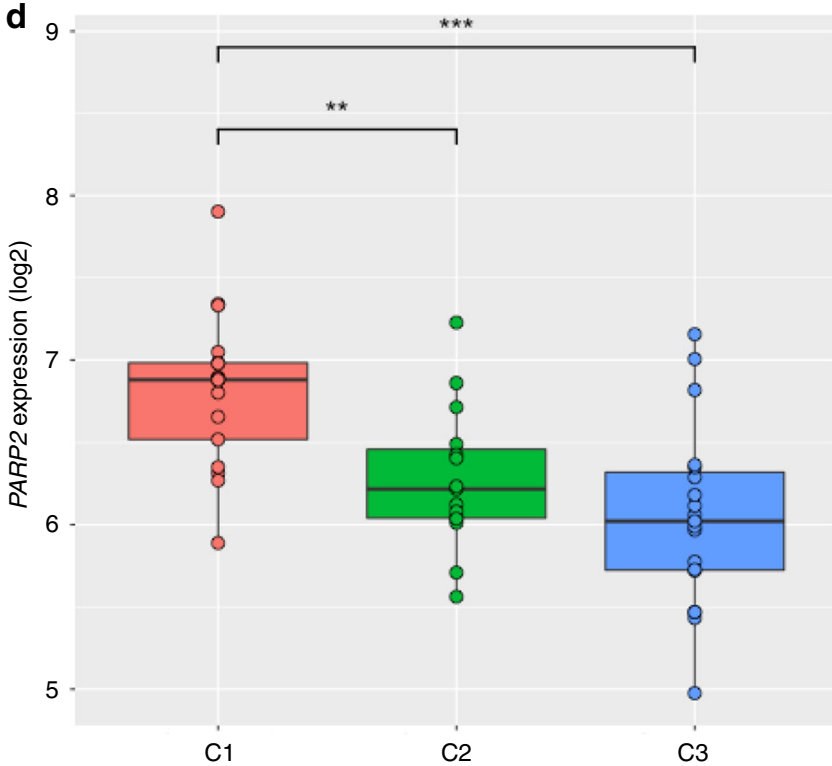

Fig. 4 Copy number alterations and immune score associations. a, b Pearson correlation of the number of deleted genes and immune scores generated from ESTIMATE for our OS cohort (a) and the TARGET cohort (b). c Boxplot of PARP2 copy number levels (in log2 level) in each of the immune infiltrate groups $\mathrm{C1}, \mathrm{C2}$, and $\mathrm{C} 3$ (as decribed in Fig. 3b). Wilcoxon rank sum test was used to determine significance. $\mathbf{d}$ Boxplot of PARP2 gene expression levels (in $\log 2$ level) in each of the immune infiltrate groups C1, C2, and C3 (as decribed in Fig. 3b). Wilcoxon rank sum test was used to determine significance.

of tumor-intrinsic adaptive immune resistance mechanisms that allow for immune evasion or lack of T-cell activation (e.g., PD-L1, CTLA4, and MDSCs). Identification of a "hot" subset of OS supports the rationale for developing a biomarker-selected approaches to future immunotherapy trials in OS. Those with lower immune infiltrate were void of most types of immune cells and associated with higher number of genes with copy number loss as well as decreased HLA expression. This corroborates previous findings that copy number alterations and aneuploidy contribute to immune suppression ${ }^{30,31}$. In fact, out of multiple genomic alterations including copy number gains, copy number loss had the highest association with immune suppression in OS. 
This suggests copy number loss may have greater impact on cellular pathways than other alterations due to permanent loss of many genes ${ }^{34}$.

These data highlight the need to pursue multiple contributors to immune suppression in OS. It is unlikely that any single approach will be effective across this patient population. Future clinical and translational studies should (1) enrich for OS patients with high immune infiltrate/immune scores who may be more likely to benefit from immune checkpoint inhibitors with differing IO approaches for those with low vs. intermediate to high immune infiltrate, (2) explore novel approaches to enhance neoantigen expression such as radiation, NMD inhibition, or intratumoral vaccines, and (3) develop rational combinations of targeted therapy and immunotherapy. For example, amongst those with intermediate to high immune infiltrate (C2 and $\mathrm{C} 3$ ) strategies that combine ICI with other IO agents or targeted therapies to mitigate the immunosuppressive genes that were expressed at higher levels in these clusters such as TGFb or the PI3K pathway warrant further exploration. Conversely, PARP2 gains and increased expression were associated with low immune infiltrate in cluster group $1(\mathrm{C} 1)$. Inhibiting PARP may render susceptibility to ICI, therefore this abundance of PARP supports the rationale for exploring combinations of PARP inhibitors and immunotherapy in OS. Ongoing translational studies from OS patients treated with immune checkpoint inhibitors may further inform the next steps in developing immunotherapy trials for this patient population ${ }^{35}$.

In conclusion, the immunogenomic landscape in OS is characterized by genomic complexity and significant disease heterogeneity. Rather than promoting a robust immune response, this genomic complexity may contribute to an immunosuppressive phenotype through multiple mechanisms that may present themselves as opportunities for novel therapeutic exploitation.

\section{Methods \\ Patients and sample collection. Collection and use of patient samples were obtained by informed consent and approved by the University of Texas MD Anderson Cancer Center Institutional Review Board. We collected all available frozen OS specimens for immunogenomic characterization: frozen resected OS primary tumors $(n=14)$, locally recurrent OS tumors $(n=13)$, lung metastases $(n$ $=27$ ) and matched adjacent normal tissues as well as matched blood $(n=2)$ from 48 pediatric and adult patients with high-grade OS. The clinical patient char- acteristics are summarized in Table 1.}

Whole genome sequencing (WGS). Genomic DNA (gDNA) was extracted with the QIAamp DNA Mini kit (Qiagen, Germantown, MD) and used for high depth paired-end whole genome sequencing. Whole genome sequencing data was generated at Baylor College of Medicine - Human Genome Sequencing Center (BCMHGSC) using established library preparation and sequencing methods. Libraries were prepared using KAPA Hyper PCR-free library reagents (KK8505, KAPA Biosystems) on Beckman robotic workstations (Biomek FX and FXp models). Briefly, DNA (500 ng) was sheared into fragments of approximately 200-600 bp using the Covaris E210 system (96-well format, Covaris, Inc. Woburn, MA) followed by purification of the fragmented DNA using AMPure XP beads. A double size selection step was employed, with different ratios of AMPure XP beads, to select a narrow size band of sheared DNA molecules for library preparation. DNA end-repair and 3'-adenylation were then performed in the same reaction followed by ligation of the barcoded adaptors to create PCR-Free libraries, and the library run on the Fragment Analyzer (Advanced Analytical Technologies, Ames, Iowa) to assess library size and presence of remaining adapter dimers. This was followed by qPCR assay using KAPA Library Quantification Kit using their SYBR ${ }^{\circledR}$ FAST qPCR Master Mix to estimate the size and quantification. WGS libraries were sequenced on the Illumina HiSeq-X instrument using the Reagent Kit v2.5 (FC-501-2501) and libraries were loaded at an average concentration of $280 \mathrm{pM}$ to generate $150 \mathrm{bp}$ paired-end reads. Unique aligned sequence in these samples varied between $209 \mathrm{~Gb}$ - $255 \mathrm{~Gb}$ per sample. The average insert sizes in these samples were $435 \mathrm{bp}$ median and $414 \mathrm{bp}$ mode. For each sample, the reads were mapped to the hg19 reference genome using BWA-MEM, followed by the downstream analyses detailed below.

Somatic point/indel mutation calling and related analysis. Somatic point variants were called from aligned WGS data using $\mathrm{MuTect}^{36}$. High quality variants were defined as those with a minimum tumor read depth of $\geq 30$, minimum matched normal read depth of $\geq 15$, and minimum alternate allele frequencies in the tumor and normal as $\geq 0.05$ and $\leq 0.01$, respectively. Kataegis were identified as those genomic regions containing eight or more consecutive mutations with an average intermutation distance of less than or equal to 1000 bp using the $\mathrm{R}$ package, ClusteredMutations ${ }^{37}$. Indel variants were called using Pindel $^{38}$. Pindel raw calls were further filtered to select for calls with score $>30$ ESP6500 and $1000 \mathrm{G}$ population minor allele frequencies $>0.01$, and not intronic. Neoantigens were predicted from the point and indel mutations using PHLAT $^{39}$ and NetMHC ${ }^{40}$.

We used two methods to identify mutation signatures in our samples. First, we used Nonnegative Matrix Factorization (NMF) to do de-novo mutation signatures discovery from our patient samples based on the method described by Alexandrov et al. $^{41}$. Then, we compared the two identified mutation signatures with the 30 published COSMIC mutation signatures (http://cancer.sanger.ac.uk/cosmic/ signatures) using the cosine similarity metric. Second, we factorized the mutation spectrum based on the 30 known COSMIC mutation signatures using the fast combinatorial non-Negative least-square algorithm ${ }^{42}$ to solve the NMF problem.

Somatic copy number calling and related analysis. Total copy number calls were derived using HMMcopy ${ }^{43}$, and $\log 2$ scores $>0.5$ were considered gains while $\log 2$ scores $<-0.5$ were considered losses. The weighted genome instability index (WGII) ${ }^{44}$ was used to quantitatively characterize portion of the genome with copy number alterations as a measure of chromosomal instability. Focal recurrent copy number alterations were identified using GISTIC $2.0^{45}$ at $95 \%$ confidence level. The $\mathrm{R}$ package, CNTools ${ }^{46}$, was used to process the segmented total copy number data into gene-level data. Allele specific copy number calls, purities, ploidies, and loss of heterozygosity of the tumor samples were derived using Sequenza ${ }^{47}$ with default parameters. Chromothripsis was detected using total copy number profiles by CTLPScanner ${ }^{48}$

Genome doubling. An in-house algorithm was developed with modifications based on previously published studies ${ }^{17,44}$ was applied to detect genome doubling in the samples. The algorithm is detailed below.

(1) Summarize absolute copy number of two alleles in chromosome arm level. $\left(N_{s}\right)$

(2) Calculate total number of aberrations (relative to diploid) for each sample

$$
N_{s}=\sum_{i=1}^{44} \sum_{j=1}^{2}\left|\mathrm{CNV}_{i, j}-1\right| \text { where } \mathrm{CNV}_{i, j} \text { is absolute copy number in arm } i \text { and } j
$$

allele for sample $s$. We only consider 22 chromosomes (44 arms).

Calculate probability of gain $\left(N_{\text {gain }}\right)$ and loss $\left(N_{\text {loss }}\right)$ for sample $s$.

$$
\begin{gathered}
P_{s, \text { gain }}=N_{\text {gain }} / N_{s} \\
P_{s, \text { loss }}=N_{\text {loss }} / N_{s}
\end{gathered}
$$

(4) Do 10,000 simulation. In each simulation for a sample, we start with 1 copy in each allele and each arm. Then we sample $N_{s}$ gain and loss based the gain and loss probabilities, and add the gain or loss to a randomly selected allele and arm.

(5) Calculate number of simulations $(M)$ in which number of arms with the major allele copy number $\geq 2$ were higher than that observed in the sample. A $p$ value for genome doubling of a sample $=M / 10,000$.

(6) A $p$-value threshold of 0.001 was used to determine if a sample went through genome doubling.

(7) Samples with $p<0.001$ and major copy number $=2$ have 1 genome doubling. Samples with $p<0.001$ and major copy number $\geq 3$ have $>1$ genome doubling.

Rearrangement calling and rearrangement signatures. Structural rearrangements were detected from aligned WGS data using BReakpoint AnalySiS (BRASS $)^{13}$. We used the list of fragile site genomic locations from Fungtammasan et al. ${ }^{49}$ to intersect with the Brass calls to identify rearrangements in the fragile sites. Expressed rearrangements identification is described in the RNA sequencing section.

The rearrangement signatures in the samples were identified using the strategy revised from the previously published studies ${ }^{13}$ to identify rearrangement signatures in the samples. First, we separated rearrangements that occurred as focal catastrophic events (clustered) or focal driver amplicons (unclustered). Nik-Zainal et al. ${ }^{13}$ used the piecewise constant fitting method to determine clustered breakpoints in each sample if the mean of inter-breakpoint distance of clustered regions are at least 10 times smaller than average inter-breakpoint distance of the whole genome. This method will be biased if majority of rearrangements are resulted from chromthripsis. Therefore, we identified clustered rearrangement breakpoints that locate in the chromothripsis regions using CTLPScanner ${ }^{48}$. Second, clustered and unclustered rearrangements are subclassified into deletions, inversions, tandem duplications, and interchromosomal translocations. Third, except interchromosomal translocations, other clustered and unclustered rearrangements are further categorized by their lengths $(1-10 \mathrm{~kb}, 10 \mathrm{~kb}-100 \mathrm{~kb}$, $100 \mathrm{~kb}-1 \mathrm{Mb}, 1 \mathrm{Mb}-10 \mathrm{Mb}$, more than $10 \mathrm{Mb}$ ). Finally, we applied Nonnegative Matrix Factorization (NMF) to the classified matrix of 32 categories of rearrangements to do de-novo rearrangement signatures discovery from our 
samples. The silhouette width-based method determined two optimal signatures. Comparisons the two identified rearrangement signatures with the 6 published signatures identified in the 560 breast cancer samples using the cosine similarity metric found the two signatures mostly correlate with the signature 2 (correlation $=0.94)$ and 4 (correlation $=0.88)$, respectively.

Telomere length. Telomere lengths of tumor and normal samples were estimated from WGS data using the TelSeq ${ }^{50}$. The tool estimates telomere length of a sample by counting the number of reads containing telomeric repeats, TTAGGG. Considering telomere length may be associated with patient age or other individual factor, we used ratio of telomere lengths between pairs of tumor and normal samples (T/N TL ratio) in our analysis.

\section{Suclonal architecture}

Consensus mutations. We used MuSE ${ }^{51}$ to call the somatic mutations based on paired tumor normal paired DNAseq data. The SNV called by MuSE are compared to MuTect. If a mutation is called by both variant caller, we define it as a consensus mutations. The we define a statistic, overlap ratio $=\frac{\text { number of consensus mutations }}{\text { number of MuTect mutations }}$, to measure the agreement between the two callers. A higher overlap ratio indicates a better agreement between the two callers. Supplemental Figure 12a shows a summary histogram of overlap ratio of the two callers.

There is high correlation between tumor purity and consensus calls (Supplemental Figure 12b) as well as the mutation number versus the overlap ratio (Supplemental Figure 12c).

Subclonal mutation reconstruction. We applied CliP (Clonal structure identification through pair-wise penalization $)^{52}$, for subclonal mutation architecture reconstruction, using the consensus mutations. Subclonal architecture reconstruction groups the somatic SNVs according to the proportion of cells in a given sample that harbor these SNVs (i.e., the Cellular Prevalences of SNVs). CliP utilizes a model based clustering approach and estimates the model parameters using a regularized maximum likelihood estimation implemented using alternating direction method of multipliers (ADMM). CliP is much faster than most existing methods, and has comparable/better performance which makes CliP ultimately suitable for large studies. Importantly. Furthermore, CliP does not require the number of clusters as input, which was required by most clustering based methods. Sequenza estimated tumor purity and copy number variant data of the 35 OS samples. Supplemental Fig. 12d shows the summary of subclonal structure of the 35 samples.

RNA sequencing and related analysis. Total RNA of samples was extracted and libraries made from cDNA using the NuGEN Ovation Ultralow Library System V2 (San Carlos, CA). For estimation of expressed neoantigens and immune therapy samples, libraries were generated using Agilent SureSelect ${ }^{\mathrm{XT}}$ RNA Direct (Agilent Technologies) (Supplemental Data 11). RNA sequencing reads of the samples were mapped to the hg19 reference genome using the STAR aligner ${ }^{53}$. For calculation of gene expression, raw count data of each gene were first obtained using with HTSeq ${ }^{54}$ and are normalized by scaling the raw library size using calcNormFactors in edgeR package in $\mathrm{R}^{55}$. Then, Voom transformation was applied to normalized counts and a linear model fit to the data for differential expression analysis using the Limma package ${ }^{56}$. Fusion transcripts were detected from RNASeq data using MapSplice ${ }^{57}$. To identified expressed rearrangements, we also integrated in-frame rearrangements and fusion transcripts to identify expressed rearrangements in each sample, whose genomic breakpoints detected from WGS data and fusion transcript junction regions detected from RNA-seq are in the same genic regions.

Immune infiltration scores were calculated from the gene expression data using several methods: ESTIMATE ${ }^{22}$, TIMER ${ }^{28}$, and cytolytic scores (calculated as the geometric mean of GZMA and PRF1 gene expression values) ${ }^{29}$. Immune infiltrate profiles of samples were generated using single sample Gene Set Enrichment Analysis (ssGSEA) enrichment scores of each immune gene list from Charoentong et al. ${ }^{27}$. Pathway analyses of differentially expressed genes between any two immune clusters were performed using Gene Set Enrichment Analysis (GSEA) ${ }^{58}$.

Genetic alterations associated with immune infiltrates. We used an integrative analysis to identity genetic alterations that are associated with immune infiltrates. We did correlation analysis of the ESTIMATE immune score respectively with gene alterations and gene expression. The product truncated method was then applied to combine $p$-values of two correlation analyses for each gene. Genes with significant combined $p$-values $(p<0.01)$ would have significant correlations of the immune score both with its genetic alterations and gene expression. We identified PARP2 whose amplifications and gene expression are both significantly negatively correlated with the ESTIMATE immune score.

Reverse phase protein array. Tumor lysates were serially diluted from undiluted to $1: 16$ and arrayed on nitrocellulose-coated slides in an $11 \times 11$ format and probed with 304 unique antibodies (Supplemental Data 12a). Of those 299 antibodies passed quality assurances and were used for further analysis. Following antibody probing of lysates on the slide, a flatbed scanner was used to produce 16-bit tiff image with subsequent spot identification and density quantification using the Array-Pro Analyzer (Meyer Instruments). SuperCurve Rx64 3.1.159 was used to derive normalized $\log 2$ values with further normalization for protein loading and transformation into linear values. We only included the data for the individual antibodies with QC Scores higher than 0.80 in the heatmap. The heatmap was generated in Cluster $3.0^{60}$ as a hierarchical cluster using Pearson Correlation and a center metric. The resulting heatmap was visualized in Treeview ${ }^{61}$. Pathway analysis was conducted as previously described. A listing of the antibodies used for each pathway analyzed is in Supplemental Data 12b).

T-cell receptor sequencing. Immunosequencing of the CDR3 regions of human TCR $\beta$ chains was performed using the ImmunoSEQ Assay (Adaptive Biotechnologies). Through the ImmunoSEQ Analyzer platform, TCRB rearrangements were extracted along with their read counts, read proportions and appropriate TCRB gene usage. T-cell clonality was defined as 1-Peilou's evenness and was calculated on productive rearrangements by:

$$
1+\left(\sum_{i}^{N} p_{i} \log _{2} p_{i} / \log _{2}(N)\right)
$$

where ${ }_{p i}$ is the proportional abundance of rearrangement $i$, and $N$ is the total number of rearrangements. Difference in T-cell density, unique rearrangements, and clonality within samples were calculated as the difference between the highest and lowest values in a tumor, expressed as a percentage of the highest value $[(\max -\min ) / \max \times 100]$. MOI is a measure of the similarity in the T-cell repertoire between samples ranging from 0 to 1 , taking into account the specific rearrangements and their respective frequencies, with an MOI of 1 being an identical T-cell repertoire. Enriched T-cell rearrangements were calculated by comparing the peripheral T-cell repertoire in patients with available blood to the tumor T-cell repertoire. Subsequent analyses were performed only on significantly enriched Tcell rearrangements within the tumor. Next, the data was split based on the rearrangements observed in each sample and the proportion of the most abundant clonotypes were calculated. Following which, clonal space homeostasis was modeled as an extension of population dynamics. A ratio of 0 to 1 was used to determine how much space was occupied by each TCR clonotype. Clonal spaces were broken down in Fig. $4 \mathrm{~b}$ as rare $(0<\mathrm{X}<1 \mathrm{e}-5)$, small $(1 \mathrm{e}-5<\mathrm{X}<1 \mathrm{e}-4)$, medium $(1 \mathrm{e}-4<\mathrm{X} 1 \mathrm{e}-3)$, large $(0.001<\mathrm{X}<0.01)$ and hyper expanded $(0.01<\mathrm{X} \leq 1)$ clones.

Immunohistochemistry. Decalcified formalin-fixed, paraffin-embedded (FFPE) blocks of osteosarcoma tissue from surgical resection specimens were retrieved from MD Anderson's institutional tumor bank. All specimens were fixed for at least $8 \mathrm{~h}$ in buffered formalin-fixed and decalcified using $10 \%$ formic acid. Slides of hematoxylin and eosin-stained sections were reviewed with a bone and soft pathologists and two tissue cores (0.6-mm diameter) were extracted from representative tumor areas of the FFPE to construct tissue microarrays. Slides of $4-\mu \mathrm{m}$ thick unstained tissue sections were prepared from the tissue microarrays of decalcified FFPE human OS specimens retrieved from the UT MD Anderson's institutional tumor bank. Immunohistochemical staining was independently scored by two pathologists who were blinded to the clinical data at the time of assessment. The list of antibodies and their dilutions are shown in the Supplemental Data 13. The correlation between the continuous biomarkers and age at diagnosis was measured by Spearman correlation coefficient. Wilcoxon rank sum test was used to compare age at diagnosis between two biomarker subgroups.

Data analysis of the TARGET project. Copy number segment and gene expression row count data of TARGET OS samples were downloaded from TARGET data matrix (https://ocg.cancer.gov/programs/target/data-matrix). The same methods used in our cohort samples were applied to process and analyze these data.

Reporting summary. Further information on research design is available in the Nature Research Reporting Summary linked to this article.

\section{Data availability}

The whole genome and RNA sequencing data will be at the European Genome-phenome Archive under the study accession number: EGAS00001003247. The RPPA data is found under the study accession number: TCPA00000004. T-cell receptor sequencing is housed at Adaptive Technologies: [10.21417/CW2020NC].

Received: 10 December 2018; Accepted: 20 January 2020; Published online: 21 February 2020

\section{References}

1. Mirabello, L., Troisi, R. J. \& Savage, S. A. Osteosarcoma incidence and survival rates from 1973 to 2004: data from the Surveillance, Epidemiology, and End Results Program. Cancer 115, 1531-1543 (2009). 
2. Bielack, S. S. et al. Prognostic factors in high-grade osteosarcoma of the extremities or trunk: an analysis of 1,702 patients treated on neoadjuvant cooperative osteosarcoma study group protocols. J. Clin. Oncol. 20, 776-790 (2002).

3. Meyers, P. A. et al. Osteogenic sarcoma with clinically detectable metastasis at initial presentation. J. Clin. Oncol. 11, 449-453 (1993).

4. Kager, L. et al. Primary metastatic osteosarcoma: presentation and outcome of patients treated on neoadjuvant Cooperative Osteosarcoma Study Group protocols. J. Clin. Oncol. 21, 2011-2018 (2003).

5. Chen, X. et al. Recurrent somatic structural variations contribute to tumorigenesis in pediatric osteosarcoma. Cell Rep. 7, 104-112 (2014).

6. Behjati, S. et al. Recurrent mutation of IGF signalling genes and distinct patterns of genomic rearrangement in osteosarcoma. Nat. Commun. 8, 15936 (2017).

7. Perry, J. A. et al. Complementary genomic approaches highlight the PI3K/ mTOR pathway as a common vulnerability in osteosarcoma. Proc. Natl. Acad. Sci. USA 111, E5564-E5573 (2014).

8. Du, X., Yang, J., Yang, D., Tian, W. \& Zhu, Z. The genetic basis for inactivation of Wnt pathway in human osteosarcoma. BMC cancer 14, 450 (2014).

9. Grignani, G. et al. Sorafenib and everolimus for patients with unresectable high-grade osteosarcoma progressing after standard treatment: a nonrandomised phase 2 clinical trial. Lancet Oncol. 16, 98-107 (2015).

10. Martin-Broto, J. et al. Gemcitabine plus sirolimus for relapsed and progressing osteosarcoma patients after standard chemotherapy: a multicenter, single-arm phase II trial of Spanish Group for Research on Sarcoma (GEIS). Ann. Oncol. 28, 2994-2999 (2017).

11. Tawbi, H. A. et al. Pembrolizumab in advanced soft-tissue sarcoma and bone sarcoma (SARC028): a multicentre, two-cohort, single-arm, open-label, phase 2 trial. Lancet Oncol. 18, 1493-1501 (2017).

12. D'Angelo, S. P. et al. Nivolumab with or without ipilimumab treatment for metastatic sarcoma (Alliance A091401): two open-label, non-comparative, randomised, phase 2 trials. Lancet Oncol. 19, 416-426 (2018).

13. Nik-Zainal, S. et al. Landscape of somatic mutations in 560 breast cancer whole-genome sequences. Nature 534, 47-54 (2016).

14. Sheltzer, J. M. et al. Aneuploidy drives genomic instability in yeast. Science 333, 1026-1030 (2011).

15. Maciejowski, J. \& de Lange, T. Telomeres in cancer: tumour suppression and genome instability. Nat. Rev. Mol. Cell Biol. 18, 175-186 (2017).

16. Gerstung, M. et al. The evolutionary history of 2,658 cancers. Nature 578, 122-128 (2020).

17. Carter, S. L. et al. Absolute quantification of somatic DNA alterations in human cancer. Nat. Biotechnol. 30, 413-421 (2012).

18. Chen, Y.-J. et al. Association of mutant TP53 with alternative lengthening of telomeres and favorable prognosis in glioma. Cancer Res. 66, 6473-6476 (2006).

19. Nabetani, A. \& Ishikawa, F. Alternative lengthening of telomeres pathway: recombination-mediated telomere maintenance mechanism in human cells. J. Biochem. 149, 5-14 (2011).

20. DeNardo, D. G., Johansson, M. \& Coussens, L. M. Immune cells as mediators of solid tumor metastasis. Cancer Metastasis Rev. 27, 11-18 (2008)

21. Rizvi, N. A. et al. Cancer immunology. Mutational landscape determines sensitivity to PD-1 blockade in non-small cell lung cancer. Science 348, 124-128 (2015).

22. Yoshihara, K. et al. Inferring tumour purity and stromal and immune cell admixture from expression data. Nat. Commun. 4, 2612 (2013).

23. O'Brien, T. D. et al. Inconsistency and features of single nucleotide variants detected in whole exome sequencing versus transcriptome sequencing: A case study in lung cancer. Methods 83, 118-127 (2015).

24. Coudray, A., Battenhouse, A. M., Bucher, P. \& Iyer, V. R. Detection and benchmarking of somatic mutations in cancer genomes using RNA-seq data. PeerJ 6, e5362 (2018)

25. Harden, J. L., Hamm, D., Gulati, N., Lowes, M. A. \& Krueger, J. G. Deep sequencing of the $\mathrm{T}$-cell receptor repertoire demonstrates polyclonal T-cell infiltrates in psoriasis. F1000Research 4, 460 (2015).

26. Chu, N. D. et al. Longitudinal immunosequencing in healthy people reveals persistent $\mathrm{T}$ cell receptors rich in highly public receptors. BMC Immunol. 20, 19 (2019).

27. Charoentong, P. et al. Pan-cancer immunogenomic analyses reveal genotypeimmunophenotype relationships and predictors of response to checkpoint blockade. Cell Rep. 18, 248-262 (2017).

28. Li, T. et al. TIMER: A web server for comprehensive analysis of tumor infiltrating immune cells. Cancer Res. 77, e108-e110 (2017).

29. Rooney, M. S., Shukla, S. A., Wu, C. J., Getz, G. \& Hacohen, N. Molecular and genetic properties of tumors associated with local immune cytolytic activity. Cell 160, 48-61 (2015).

30. Davoli, T., Uno, H., Wooten, E. C. \& Elledge, S. J. Tumor aneuploidy correlates with markers of immune evasion and with reduced response to immunotherapy. Science 355, eaaf8399 (2017).
31. Roh, W. et al. Integrated molecular analysis of tumor biopsies on sequential CTLA-4 and PD-1 blockade reveals markers of response and resistance. Sci. Transl. Med. 9, eaah3560 (2017).

32. Flynn, R. L. et al. Alternative lengthening of telomeres renders cancer cells hypersensitive to ATR inhibitors. Science 347, 273-277 (2015).

33. Ma, X. et al. Pan-cancer genome and transcriptome analyses of 1,699 paediatric leukaemias and solid tumours. Nature 555, 371 (2018).

34. Mishra, S. \& Whetstine, J. R. Different facets of copy number changes: permanent, transient, and adaptive. Mol. Cell Biol. 36, 1050-1063 (2016).

35. Burgess, M. A. et al. Multicenter phase II study of pembrolizumab (P) in advanced soft tissue (STS) and bone sarcomas (BS): Final results of SARC028 and biomarker analyses. J. Clin. Oncol. 35, 11008-11008 (2017).

36. Cibulskis, K. et al. Sensitive detection of somatic point mutations in impure and heterogeneous cancer samples. Nat. Biotechnol. 31, 213-219 (2013).

37. Location and Visualization of Clustered Somatic Mutations v. 1.0.1 (CRAN, 2016).

38. Ye, K., Schulz, M. H., Long, Q., Apweiler, R. \& Ning, Z. Pindel: a pattern growth approach to detect break points of large deletions and medium sized insertions from paired-end short reads. Bioinformatics 25, 2865-2871 (2009).

39. Bai, Y., Wang, D. \& Fury, W. PHLAT: inference of high-resolution HLA types from RNA and whole exome sequencing. Methods Mol. Biol. 1802, 193-201 (2018).

40. Lundegaard, C. et al. NetMHC-3.0: accurate web accessible predictions of human, mouse and monkey MHC class I affinities for peptides of length 8-11. Nucleic Acids Res. 36, W509-W512 (2008).

41. Alexandrov, L. B. et al. Signatures of mutational processes in human cancer Nature 500, 415-421 (2013)

42. Van Benthem, M. H., Keenan, M. R. \& Haaland, D. M. Application of equality constraints on variables during alternating least squares procedures. $J$. Chemometrics 16, 613-622 (2002).

43. HMMcopy: Copy number prediction with correction for GC and mappability bias for HTS data. v. R package version 1.22.0. (Bioconductor, 2016).

44. Dewhurst, S. M. et al. Tolerance of whole-genome doubling propagates chromosomal instability and accelerates cancer genome evolution. Cancer Disco. 4, 175-185 (2014).

45. Mermel, C. H. et al. GISTIC2.0 facilitates sensitive and confident localization of the targets of focal somatic copy-number alteration in human cancers. Genome Biol. 12, R41 (2011).

46. CNTools: Convert segment data into a region by sample matrix to allow for other high level computational analyses. v. R package version 1.36.0. (Bioconductor, 2018)

47. Favero, F. et al. Sequenza: allele-specific copy number and mutation profiles from tumor sequencing data. Ann. Oncol. 26, 64-70 (2015).

48. Yang, J. et al. CTLPScanner: a web server for chromothripsis-like pattern detection. Nucleic Acids Res. 44, W252-W258 (2016).

49. Fungtammasan, A., Walsh, E., Chiaromonte, F., Eckert, K. A. \& Makova, K. D. A genome-wide analysis of common fragile sites: what features determine chromosomal instability in the human genome? Genome Res. 22, 993-1005 (2012).

50. Ding, Z. et al. Estimating telomere length from whole genome sequence data Nucleic Acids Res. 42, e75 (2014).

51. Fan, Y. et al. MuSE: accounting for tumor heterogeneity using a samplespecific error model improves sensitivity and specificity in mutation calling from sequencing data. Genome Biol. 17, 178 (2016).

52. Dentro, S. C. et al. Portraits of genetic intra-tumour heterogeneity and subclonal selection across cancer types. Preprint at: https://www.biorxiv.org/ content/10.1101/312041v4 (2018)

53. Dobin, A. et al. STAR: ultrafast universal RNA-seq aligner. Bioinformatics 29 (15-21 (2013)

54. Anders, S., Pyl, P. T. \& Huber, W. HTSeq-a Python framework to work with high-throughput sequencing data. Bioinformatics 31, 166-169 (2015).

55. edgeR (Bioconductor, 2016).

56. Smyth, G. K. Limma: linear models for microarray data. 397-420 (Springer, 2005).

57. Wang, K. et al. MapSplice: accurate mapping of RNA-seq reads for splice junction discovery. Nucleic Acids Res. 38, e178 (2010).

58. Mootha, V. K. et al. PGC-1alpha-responsive genes involved in oxidative phosphorylation are coordinately downregulated in human diabetes. Nat. Genet. 34, 267-273 (2003).

59. SuperCurve v. 1.5.15 (R-Forge, 2015).

60. de Hoon, M. J., Imoto, S., Nolan, J. \& Miyano, S. Open source clustering software. Bioinformatics 20, 1453-1454 (2004)

61. Page, R. D. TreeView: an application to display phylogenetic trees on personal computers. Comput. Appl. Biosci. 12, 357-358 (1996). 


\section{Acknowledgements}

Sequencing support was provided by Cancer Genomics Laboratories and Baylor Human Genome Center. Bioinformatics pipeline support was provided by John Zhang, Chang-Jiun $\mathrm{Wu}$, and Xingzhi Song. Alijunaid Alhussain and Ruth Alejo Salazar conducted immunostaining scoring. The results shown here are in part based upon data generated by the TCGA Research Network: http://cancergenome.nih.gov/. We graciously acknowledge International Cancer Genome Consortium (ICGC), The Cancer Genome Atlas (TCGA) and Therapeutically Applicable Research to Generate Effective Treatments (TARGET) (https://ocg. cancer.gov/programs/target), including specimen donors and research groups for their work. This work was supported by Triumph Over Kid Cancer Foundation (to V.L)., A Shelter for Cancer Families (ASCF), formerly Amshwand Sarcoma Cancer Foundation (to the Sarcoma Oncology Group), QuadW Foundation (to the Sarcoma Oncology Group), Cancer Prevention Research Institute of Texas (R120501 to P.A.F., RP170067 to A.M. and Y.Z.), and Welch Foundation's Robert A. Welch Distinguished University Chair Award (G-0040 to P.A.F.).

\section{Author contributions}

H.B., A.L., N.D., V.L., and P.A.F. designed the study. J.A.L., S.A., and N.D. completed clinical annotation. W.L.W., D.I., and A.L. processed the samples. W.L.W. and A.L. performed histopathological analyses. C.W., S.C., A.R., Y.Z., A.M., J.R., and W.W. performed bioinformatics analyses. C.H.L. and H.L. performed biostatistical analysis for the immunohistochemistry results. Z.J. and H.B. conducted the RPPA analyses. G.M. developed the RPPA immune signature. S.P., N.D., R.S.B., N.S. and A.C. provided specimens and clinical interpretations. C.W., H.B., J.A.L., W.W., and P.A.F. analyzed all data. H.B., C.W., J.A.L., C.W., W.W., R.G., P.H., A.L., N.D., V.L. and P.A.F. interpreted the data. H.B., J.A.L., C.W., and P.A.F. wrote the manuscript. All authors read and approved the final manuscript.

\section{Competing Interests}

The authors declare no competing interests.

\section{Additional information}

Supplementary information is available for this paper at https://doi.org/10.1038/s41467020-14646-w.

Correspondence and requests for materials should be addressed to P.A.F.

Peer review information Nature Communications thanks David Thomas and the other, anonymous, reviewer(s) for their contribution to the peer review of this work.

Reprints and permission information is available at http://www.nature.com/reprints

Publisher's note Springer Nature remains neutral with regard to jurisdictional claims in published maps and institutional affiliations.

(c) (i) Open Access This article is licensed under a Creative Commons Attribution 4.0 International License, which permits use, sharing adaptation, distribution and reproduction in any medium or format, as long as you give appropriate credit to the original author(s) and the source, provide a link to the Creative Commons license, and indicate if changes were made. The images or other third party material in this article are included in the article's Creative Commons license, unless indicated otherwise in a credit line to the material. If material is not included in the article's Creative Commons license and your intended use is not permitted by statutory regulation or exceeds the permitted use, you will need to obtain permission directly from the copyright holder. To view a copy of this license, visit http://creativecommons.org/ licenses/by/4.0/.

(C) The Author(s) 2020 